\title{
Combined Use of Two Biocontrol Agents with Different Biocontrol Mechanisms Most Likely Results in Less Than Expected Efficacy in Controlling Foliar Pathogens Under Fluctuating Conditions: A Modeling Study
}

\author{
X.-M. Xu and M. J. Jeger
}

First author: State Key Laboratory of Crop Stress Biology in Arid Areas and College of Plant Protection, Northwest A\&F University, Yangling, Shaanxi 712100, China; and Pest and Pathogen Ecology for Sustainable Crop Management, East Malling Research, New Road, East Malling, ME19 6BJ, UK; and second author: Division of Ecology and Evolution and Centre for Environmental Policy, Imperial College London, Silwood Park Campus, Ascot, SL5 7PY, UK.

Accepted for publication 13 October 2012.

\begin{abstract}
Xu, X.-M., and Jeger, M. J. 2013. Combined use of two biocontrol agents with different biocontrol mechanisms most likely results in less than expected efficacy in controlling foliar pathogens under fluctuating conditions: A modeling study. Phytopathology 103:108-116.

Effective use of biocontrol agents (BCAs) is a potentially important component of sustainable agriculture; recently, there has been a trend for combined use of several BCAs, with an expectation of synergistic interactions among them. A previous numerical study suggested that, under homogenous conditions in which two BCAs occupied the same host tissue as the pathogen, combined use of two BCAs with different biocontrol mechanisms resulted, in most cases, in efficacies similar to using the more efficacious one alone; this result is consistent with published experimental results. The present study investigates whether combined use of a mycoparasitic and a competitive BCA leads to greater efficacy

BCAs. Within the range of parameter values considered, combined use of two BCAs is shown to be less effective than that expected under the assumption of Bliss independence, and to result in a level of efficacy similar to that achieved by the more efficacious component used alone, indicating antagonistic interactions between the two BCAs. Nevertheless, combined use of two BCAs resulted in a slightly longer delay in epidemic development than did individual use of BCAs. Stochastic variability in simulated hourly temperatures did not result in a high level of variability in efficacy among replicates; nevertheless, the among-replicate variability appeared to be greater for the combined use of BCAs than for individual $\mathrm{BCAs}$ used alone. In contrast, there were greater effects of varying BCAtemperature relationships and application time (reflected in the temperature profile) on efficacy, suggesting the importance of characterizing the relationship between BCA activity and environmental conditions in future research.
\end{abstract} than that expected when the model is modified to allow for fluctuating temperature regimes and the effects of temperature on the pathogen and

Effective use of biocontrol agents (BCAs) is a potentially important component of sustainable agriculture. The ecological processes determining the success or otherwise of biocontrol are complex and it is perhaps understandable that success in biocontrol of plant diseases in field crops has been limited (2), most success being achieved in greenhouse cultivation $(6,15)$, where conditions are highly controllable.

To improve efficacies achieved through BCA application, there has recently been increasing interest in studying the efficacy of mixtures of BCAs. A range of biocontrol mechanisms may operate in mixed BCA populations $(5,7,8,20)$. The relative significance of each mechanism is, in many cases, unknown but expected to vary with circumstances. In mixed BCA populations, there may also be both direct and indirect interactions between different BCA populations. It has been suggested that combined use of BCAs may have two potential benefits (i.e., synergy in control efficacies and reduced variability in the efficacy achieved). However, a recent review of all published evidence on the combined use of BCAs showed that, contrary to the frequent claim of synergy, most published studies instead showed antagonistic

Corresponding author: X.-M. Xu; E-mail address: xiangming.xu@emr.ac.uk

http://dx.doi.org/10.1094/PHYTO-07-12-0167-R

(c) 2013 The American Phytopathological Society
Additional keyword: stochasticity. interactions among BCAs (24). This apparent contradiction resulted from the incorrect definition of "synergy" in many of the published studies. There is, as yet, insufficient evidence to either support or refute the second advantage claimed for the combined use of BCAs (i.e., reduced variability) (8).

Recently, a mean-field deterministic model for biocontrol of foliar diseases was developed and used to assess the efficacy in relation to individual biocontrol mechanisms in a single BCA (9), multiple mechanisms in one BCA (27), and combined use of two BCAs, each with a single mechanism (25). These modeling results suggest that combining mechanisms within a single BCA is generally the most effective in reducing disease development (27) but that combined use of two BCAs with a single but different mechanism would rarely result in synergy by more likely antagonistic interactions among BCAs (25), in agreement with most published experimental results (24). The generic model underlying each of these studies assumes homogenous mixing between BCAs and pathogen populations as well as homogeneous environmental conditions. Although each of these assumptions would always be violated to a varying degree in practice, the modeling results provide a baseline from which we can compare biocontrol outcomes under heterogeneous conditions. It is not clear to what extent the conclusions on the combined use of BCAs drawn from this mean-field deterministic model are also valid under heterogeneous conditions. This is particularly relevant because the lack of biocontrol success in practice is often attributed to the lack of 
our ability to predict the outcome of the interactions between a $\mathrm{BCA}$, the pathogen, the crop, the resident microflora, and the environment $(19,20)$.

In this study, the previous mean-field deterministic model of combined use of two BCAs (25) was modified to take into account the effects of fluctuating conditions on the BCA and pathogen development. The focus was to determine whether combined use of two BCAs, each with a single but different biocontrol mechanism (mycoparasitism or competition), is also more likely to result in antagonistic interactions among BCAs under fluctuating conditions, affecting biocontrol efficacy.

\section{THEORY AND APPROACHES}

Model description. Numerical simulations were used to study the effects of fluctuating conditions on the biocontrol efficacy in relation to multiple mechanisms in one BCA or combined use of two BCAs. The present model was based on the generic model developed by Jeger et al. (9), as modified for inundative application by $\mathrm{Xu}$ et al. (27). In this generic model, a standard susceptible-infected-removed model for host-pathogen dynamics was coupled with one for the pathogen-BCA dynamics without considering spatial heterogeneity. The previous study showed that a BCA combining both mycoparasitism and competition is generally most effective, although it still more likely to result in antagonistic interactions, and that combined use of a mycoparasitic and a competitive BCA results in complex damped-cyclic epidemics (25). Thus, the present study focused on the combined use of this particular pair of BCAs under temporal fluctuating conditions in a spatially homogenous context. For this combination, the original model becomes simpler because the category of healthy resistant host tissue, together with the four parameters related to induced resistance, is no longer necessary.

Host tissue was divided into the following mutually exclusive categories: healthy susceptible $\left(H_{s}\right)$, infectious disease $(I)$, removed disease (post infectious) $(R)$, and area occupied by BCA $\left(H_{b}\right) . H_{b}$ is subdivided into two mutually exclusive parts, indicated by subscript 1 or 2 . Thus, $H_{b l}$ is the host tissue colonized by BCA1, and $H_{b 2}$ the host tissue colonized by BCA2. The model assumes that two BCAs occupy the same type of host tissues as the pathogen. Furthermore, the two BCAs were assumed not to interact with each other directly, apart from occupying same host type. The full model is then:

$$
\begin{aligned}
& {\left[d H_{s} / d t\right]=-b I H_{s}-c_{11} H_{b 1} H_{s}-c_{12} H_{b 2} H_{s}+f_{1} H_{b 1}+f_{2} H_{b 2}} \\
& {\left[d H_{b 1} / d t\right]=H_{b 1}\left[c_{11} H_{s}+c_{21}\left(1-H_{s}-H_{b 1}-H_{b 2}\right)-f_{1}\right]} \\
& {\left[d H_{b 2} / d t\right]=H_{b 2}\left[c_{12} H_{s}+c_{22}\left(1-H_{s}-H_{b 1}-H_{b 2}\right)-f_{2}\right]} \\
& {[d I / d t]=I\left(b H_{s}-c_{21} H_{b 1}-c_{22} H_{b 2}-h\right)} \\
& {[d R / d t]=h I-c_{21} R H_{b 1}-c_{22} R H_{b 2}}
\end{aligned}
$$

The sum of the five equations is zero (i.e., a constant host population size). Descriptions for each model parameter are given in Table 1. For the colonization rates, $c_{11}\left(c_{12}\right)$ refers to the colonization rate of healthy host tissue $H_{s}$ by BCA1 (BCA2) and $c_{2 I}$ $\left(c_{22}\right)$ refers to the colonization rate of diseased host tissue $I$ and $R$ by BCA1 (BCA2). Mycoparasitism and competition biocontrol mechanisms are characterized by higher $c_{21}\left(c_{22}\right)$ or $c_{11}\left(c_{12}\right)$ values, respectively. Thus, if a particular BCA has higher $c_{11}\left(c_{12}\right)$ than $c_{21}\left(c_{22}\right)$, with $c_{21}\left(c_{22}\right)$ close to zero, then it is classified as a competitive BCA; equally, if a particular BCA has higher $c_{21}\left(c_{22}\right)$ than $c_{11}\left(c_{12}\right)$, with $c_{11}\left(c_{12}\right)$ close to zero, then it is classified as a mycoparasitic BCA; finally, if a particular BCA has a $c_{11}\left(c_{12}\right)$ value similar to $c_{21}\left(c_{22}\right)$, which are not close to zero, it is said to have both mycoparasitic and competition mechanisms. Dead BCA-occupied tissues (the mortality is parameterized as $f$ ) return to susceptible tissue, which effectively simulates host growth. The pathogen infects healthy host tissues at a rate of $b$ with a loss of infectiousness rate of $h$.

To study this particular biocontrol system under fluctuating conditions, key model $\mathrm{BCA}\left(c_{11}, c_{12}, c_{21}\right.$, and $\left.c_{22}\right)$ and pathogen $(b$ and $h$ ) parameters were assumed to depend on a variable that is temporally fluctuating. For simplicity, temperature $(T)\left({ }^{\circ} \mathrm{C}\right)$ was assumed to be the only variable driving the biocontrol system in this study. We first simulated hourly fluctuating temperatures; next, calculated individual model parameter values at each hour, assuming a certain relationship with temperature; and, finally, used the derived parameter values to numerically integrate the set of differential equations 1 a to $1 \mathrm{e}$.

Simulating fluctuating temperatures at hourly intervals. The annual pattern of daily mean temperatures was determined from daily mean temperatures from 1996 to 2011 at East Malling Research recorded by an automatic weather station (Campbell Scientific, Shepshed, Leics, UK); this pattern can be well described by

$$
\overline{T_{t}}=4.245+14.066 \exp \left(-\frac{(t-206.559)^{2}}{5123.696}\right)
$$

Equation 2 accounted for $\approx 99.1 \%$ of the total variance in the observed temperature data. Similarly, the extent of daily temperature fluctuation during the same period (i.e., the difference between daily maximum and minimum temperatures) can be described by

$$
\overline{A_{t}}=2.833+7.214 \exp \left(-\frac{(t-186.08)^{2}}{14689.44}\right)
$$

Equation 3 accounted for $\approx 94.0 \%$ of the total variance in the observed data. In both equations 2 and $3, t$ is the number of days from 1 January (i.e., for 1 and 2 January, $t$ is 1 and 2, respectively). The annual pattern in daily mean temperature and daily temperature fluctuation as described by the two models is shown in Figure 1A.

Hourly temperature at a particular hour ( $m$, from 1 to 24 ) of a particular day ( $t$, from 1 to 365 ) was then simulated using the

TABLE 1. Default values of model parameters describing rates of changes of several processes in a plant pathogen biocontrol system ${ }^{\mathrm{a}}$

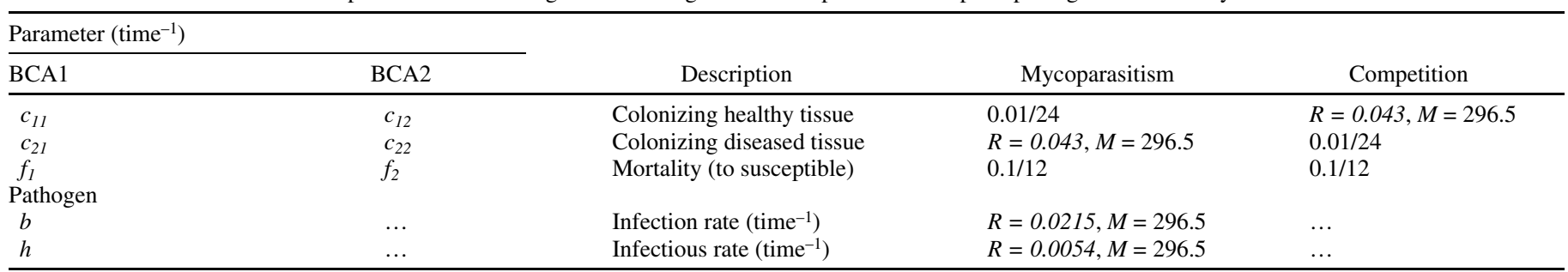

${ }^{a}$ Each set of parameter values represents a biocontrol agent (BCA) with a single main biocontrol mechanism, as specified. Ea,ch key parameter describes a single biocontrol mechanism and the two pathogen parameters are related to temperature via a thermodynamic model (equation 6), in which two parameters $(R$ and $M$ ) jointly determine $\mathrm{BCA}$ and pathogen development rates. 
following simple sine-wave model:

$$
T_{t . m}=\bar{T}_{t}+\frac{A_{t}}{2} \sin \left(\frac{m}{12} \pi\right)
$$

Finally, a random component was added to equation 4 to simulate stochasticity in hourly temperatures, i.e.,

$$
T_{t . m}=\bar{T}_{t}+\frac{A_{t}}{2} \sin \left(\frac{m}{12} \pi\right)+\varepsilon
$$

where $\varepsilon$ is a normally distributed random variable $(N[0,4])$. The variance of the random component was based on the residuals of a time-series model fitted to the hourly temperatures recorded at East Malling Research in 2010 and 2011. Several values of this variance were used $(2.25,4.0$, and 6.25$)$ but the results were similar and, hence, only the results for the variance of 4.0 are presented.

BCA and pathogen development in relation to temperature. Both BCAs and the pathogen were assumed to have the highest developmental rate at some intermediate temperatures. To model the effect of fluctuating temperature on biocontrol, the relationships of $c_{11}\left(c_{12}\right)$ (for a competitive BCA) and $c_{21}\left(c_{22}\right)$ (for a mycoparasitic BCA) with temperature were assumed to follow a thermodynamic model:

$$
r=\frac{R(T+273.2) \exp \left(20\left(1-\frac{298}{T-273.2}\right)\right)}{298\left(1+\exp \left(174\left(1-\frac{M}{T+273.2}\right)\right)\right)}
$$

where $r$ [one of model parameters in equation $1-c_{21}\left(c_{22}\right)$ and $c_{11}$ $\left.\left(c_{12}\right)\right]$ is the development rate (per hour), $M$ determines the optimum temperature, and $R$ determines the magnitude of the development rate. This model represents a typical relationship of biological organisms with temperature: developmental rate is greatest in intermediate temperatures and decreases to zero when temperature moves further away from the optimum $(21,23)$.

In the present study, three different sets of thermodynamic model parameters were used to represent $\mathrm{BCA}$ development at three optimum temperatures $\left(15,20\right.$, and $\left.25^{\circ} \mathrm{C}\right) . M$ was set to $291.4,296.5$, and 301.6 to represent optimum development at 15 , 20 , and $25^{\circ} \mathrm{C}$, respectively. In order to ensure that the maximum developmental rate was similar at the three optimum temperatures, $R$ was set to $0.0624,0.043$, and 0.03 at the optimum of 15 ,
20 , and $25^{\circ} \mathrm{C}$, respectively. These three rate-temperature relationships are shown in Figure 1B.

Because the study focus was on studying the relative relationship of pathogen and BCA development with temperature, only a single set of parameters was used for the pathogen parameters: $b$ (pathogen infection) and $h$ (pathogen infectious rate) were related to temperature as described in equation 6 . Both $b$ and $h$ were assumed to have an optimum temperature at $20^{\circ} \mathrm{C}(M=296.5)$; for $b, R=0.0215$ and, for $h, R=0.0054$ (i.e., $b$ is four times $h$ at any given temperature). Therefore, all three possible scenarios for the relative pathogen and $\mathrm{BCA}$ relationship with temperature were covered: temperatures suitable for pathogen development was lower than, equal to, or greater than for BCA development. If temperature was $<0.0^{\circ} \mathrm{C}, c_{21}\left(c_{22}\right), c_{11}\left(c_{12}\right)$, and $b$ were set to zero but $h$ was still estimated from equation 6 .

Simulation studies. Numerical studies were divided into two parts: evaluating the efficacy in relation to a range of model parameters and investigating the effect of stochasticity in temperature on biocontrol. The fourth Runge-Kutta numerical integration method (16) was implemented in the Delphi programming language to integrate equation 1 at an hourly step. The Delphi integration algorithm was checked for its accuracy by comparing its outputs against those from Maple 16 for the mean-field model over a range of parameter values.

Application time. All simulations started on one of the three dates (day 1 [1 January], 91 [1 April], and 152 [1 July]) and terminated at the end of day 365 . These three times were chosen in order to have biocontrol systems initiated in a wide range of temperatures, although epidemics are rarely initiated in early January. An epidemic was initiated with a 0.001 level of disease that was just at the point of entering the infectious period (i.e. $I_{0}=0.001$ ). BCA was assumed to be applied on the same day when the simulation initiated. When two BCAs were used in combination, they were assumed to be introduced within a short interval, ignoring the order of their applications, and could effectively be considered to be introduced at the same time for the present modeling study. Only a single spray coverage was considered because the previous study (27) showed that the effect of spray coverage of 50 to $90 \%$ on biocontrol was small. The coverage of a single BCA application was assumed to be $50 \%$ and, hence, the total coverage of combined use of two BCAs under the assumption of independence with overlapping coverage was $75 \%[=50 \%+$ $(100-50 \%) \times 50 \%$ ]. Thus, $37.5 \%$ of 0.999 healthy susceptible host tissue was allocated to each of the two BCAs.
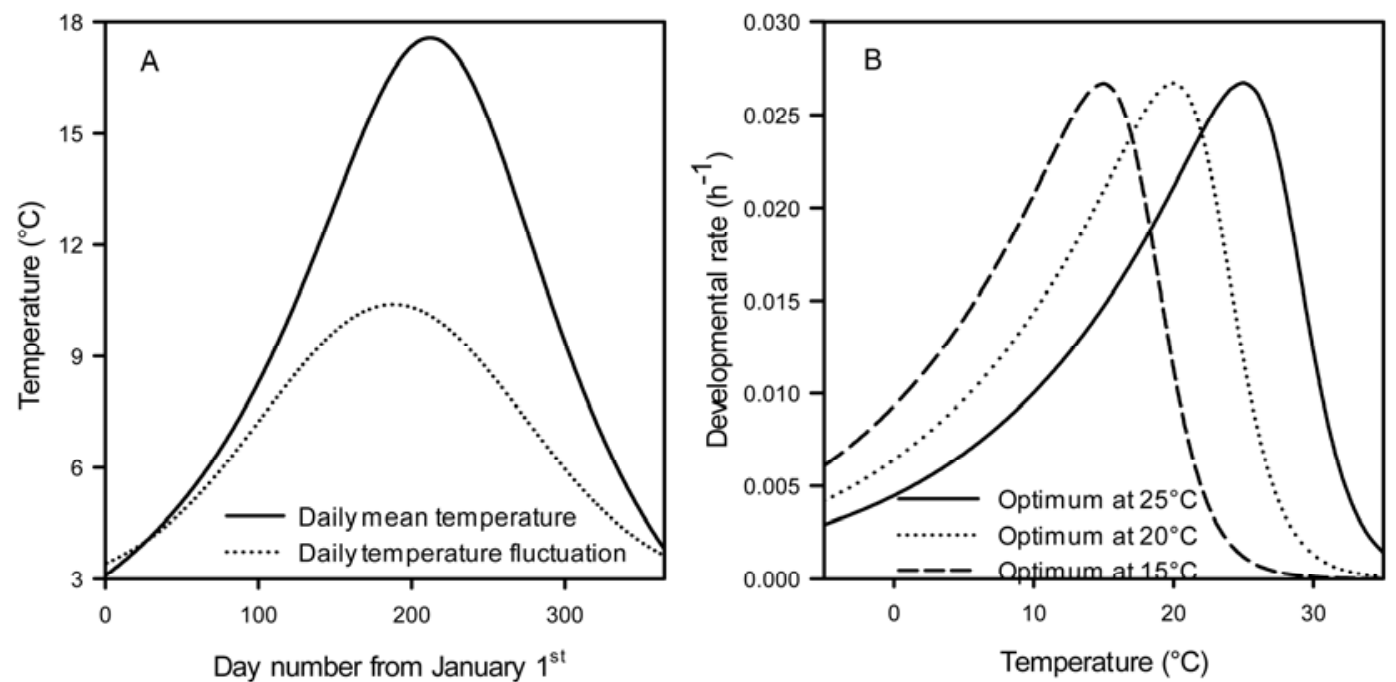

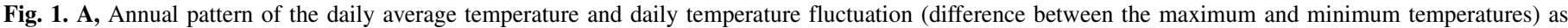

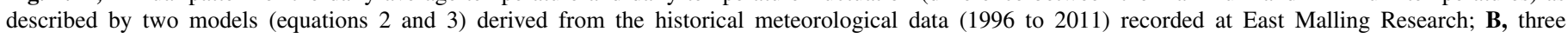
developmental rate-temperature relationships (equation 6) with the optimum at 15,20 , and $25^{\circ} \mathrm{C}$, respectively. 
BCA application scenarios. For a biocontrol system with a single BCA with either mycoparasitism or competition as its biocontrol mechanism, three optimum temperatures for $c_{22}$ (mycoparasitism) or $c_{11}$ (competition) were assumed $\left(15,20\right.$, and $25^{\circ} \mathrm{C}$ ), thus giving six scenarios of applying an individual BCA (with one mechanism only) alone. There were nine different scenarios of combined use of the two BCAs: three optimum temperatures for the mycoparasitic BCA combined with three optimum temperatures for the competitive BCA. Similarly, there were nine corresponding scenarios of applying a single BCA that had both the mechanisms, with the respective model parameters the same as in each of the two individual BCAs. Therefore, there was a total of $24(9+9+6)$ different BCA application scenarios for a given combination of model parameters, which were numerically integrated under a given simulated series of chronological temperature. In addition, whenever appropriate, a pathogen-only model was run as the basis for comparison.

Evaluating model parameters. Numerical evaluations were first conducted to investigate the efficacy of combined use of two BCAs relative to individual BCAs over a range of model biocontrol parameters $\left[c_{21}\left(c_{22}\right)\right.$ or $c_{11}\left(c_{12}\right)$ via the parameter $R$ in equation 6]. For both $c_{21}\left(c_{22}\right)$ or $c_{11}\left(c_{12}\right), 21$ different $R$ values were evaluated (i.e., ranging from 0.02 to 0.04 at an increment of 0.001 for the optimum at $25^{\circ} \mathrm{C}$, from 0.03 to 0.06 at an increment of 0.0015 for the optimum at $20^{\circ} \mathrm{C}$, and from 0.04 to 0.08 at an increment of 0.002 for the optimum at $15^{\circ} \mathrm{C}$ ). All other model parameters were set to the default values (Table 1). For a BCA that combined the two mechanisms, the value of each relevant parameter was given the same value as that for the BCA with only one mechanism. Only a single simulated series of hourly temperatures obtained by equation 4 (i.e., without the random component) was used to drive the pathogen-BCA model to evaluate the effects of model parameters on biocontrol efficacy under various BCA application scenarios.

Stochastic variation in temperature. In total, 50 different sets of hourly temperatures over a period of 365 days were simulated (equation 5) to drive the pathogen-BCA model. The same set of temperature values were used to drive the 24 different BCA application scenarios as well as the pathogen-only model. Only a single set of model parameter values was used to study the effects of stochastic variation on biocontrol; $R$ was set to $0.0624,0.43$, and 0.03 for the optimum at 15,20 , and $25^{\circ} \mathrm{C}$, respectively.

Data analysis. For each simulated disease dynamic, area under the disease progress curve (AUDPC) was calculated for the entire simulation period (365 days). Biocontrol efficacy was then estimated as $E_{b}=\left[\left(A U D P C_{P}-A U D P C_{B}\right) \times 100\right] / A U D P C_{P}$, where $A U D P C_{P}$ and $A U D P C_{B}$ are the AUDPC values for the simulated epidemics without and with BCAs, respectively. The expected biocontrol efficacy for combined use of two BCAs was estimated based on the Bliss independence (1) (i.e., $E_{M}=E_{1}+E_{2}-E_{1} \times E_{2}$, where $E_{1}$ and $E_{2}$ are the efficacy of the two individual BCAs when applied alone under the same conditions. In addition, the number of days it took to reach $10 \%$ total disease $\left(\right.$ Day $\left._{10}\right)$ for the first time was recorded for each simulation. The efficacy and Day $_{10}$ of the better-performing BCA were also compared with those from combined use of the two BCAs.

For the data on the evaluation of model parameters, a four-way analysis of variance was used to compare epidemic variables (efficacy and $\mathrm{Day}_{10}$ ) from the combined use of two BCAs with the expected efficacy and those of the better-component BCA. The four factors were (i) nine different combinations of two BCAs, (ii) 21 sets of parameter values, (iii) three application times (model start times), and (iv) the comparison of combined use with the expected or the better-component BCA. For the data on the temperature stochasticity, a linear mixed model was used, in which the nine different BCA combinations, three application times, and comparison of the combined use with the expected or the better-component BCA were treated as fixed factors and the 50 sets of temperature values was treated as a random factor. In all analyses, efficacy data were arcsine transformed, and Day ${ }_{10}$ values were square root transformed before analysis. For the stochasticity data set, the variance in the efficacy among the 50 replicate runs was compared between individual and combined use of BCAs.

\section{RESULTS}

Constant versus fluctuating temperature. Annual simulated mean temperature was $9.2^{\circ} \mathrm{C}$ based on equation 2. Examples of simulated fluctuating hourly temperatures for a period of 48 consecutive hours with or without the random component are given in Figure 2. Because temperature is much lower in January, the simulated hourly temperatures appeared to deviate more from the diurnal pattern in January than in the other two months.

Considerable differences in disease development may result regarding whether fluctuating or constant temperatures were used to drive the model (Fig. 3), and such differences also depended on the biocontrol mechanism involved. Thus, in the example where both mycoparasitic and competitive BCAs had optima at $25^{\circ} \mathrm{C}$ and were applied on 1 January, disease development in the presence of a competitive BCA was considerably delayed under constant temperature (Fig. 3B) but the opposite was true in the presence of a mycoparasitic BCA. When a mycoparasitic BCA was used alone or in combination with another BCA, disease development exhibited oscillations under both constant and fluctuating temperatures (Fig. 3C).

Model parameters. When co-introduced with the pathogen at the level of 0.001 incidence, biocontrol efficacy resulting from application of individual BCAs or joint use of two BCAs varied greatly with the BCA optimum temperatures, application time, and parameter values. The differences in the epidemic development of the joint use of BCAs with the expected or the bettercomponent BCA generally decreased with late application (model start) time and with increased optimum temperatures for the BCA


Fig. 2. Three examples of simulated hourly temperatures with (equation 5) or without (equation 4) the random component for a 48-h period in A, January; B, May; and $\mathbf{C}$, August. 
(Fig. 4). Overall, the main effect of application time was relatively small when compared with the other simulation variables, except for a few specific combinations of BCAs (e.g., CM25/M15 and C25/M20) (Fig. 4A), but its interaction with the other simulation variables was large for Day ${ }_{10}$. Thus, only data from the application on day 91 are presented hereafter, unless specified otherwise, because the focus was on the comparison of combined use of BCAs with individual use.

Efficacy from the combined use of two BCAs generally increased with increasing model parameter values (Fig. 5). Nevertheless, there were small reductions in biocontrol efficacies associated with increasing parameter values, mostly occurring for small parameter values (Fig. 5G to I). The shape and magnitude of the increase in the efficacy with increasing parameter values varied with particular combinations of BCAs. Low efficacies resulted from combined use of two BCAs, with the competitive $\mathrm{BCA}$ having the optimum temperature at $25^{\circ} \mathrm{C}$ (Fig. $5 \mathrm{G}$ to I versus the others). In contrast, the effect of varying optimum temperature for mycoparasitic activity was relatively small and increased with increasing optimum temperature for the competi-

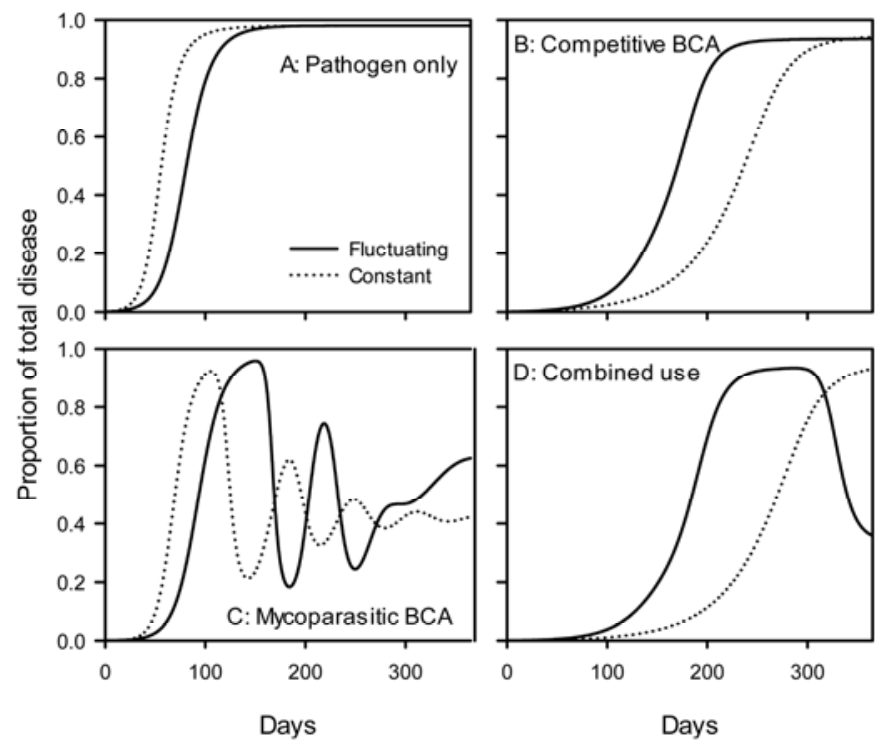

Fig. 3. Examples of simulated epidemic development in the presence or absence of one biocontrol agent (BCA) or two BCAs under fluctuating or constant temperature conditions. The average temperature under fluctuating conditions is equal to the constant temperature $\left(9.2^{\circ} \mathrm{C}\right)$ that was used to drive the model. The model started on 1 January with the default model parameters (Table 1) except that both competitive and mycoparasitic BCAs have their optimum temperature at $15^{\circ} \mathrm{C}$. tive BCA; increasing optimum temperature for mycoparasitic activity generally led to decreased efficacies (Fig. 5).

In all cases, a single BCA with two biocontrol mechanisms was more effective than a single BCA with one mechanism or combined use of two BCAs, each with one of the two mechanisms (data not shown); indeed, in most cases, the efficacy was nearly $100 \%$. Over the entire range of parameter values investigated, the efficacy resulting from combined use of two BCAs was slightly greater than the more efficacious one of the two, especially for combinations involving a competitive BCA with an optimum at $25^{\circ} \mathrm{C}$ (Fig. 5G to I). In a few specific combinations of BCAs, over a narrow range of parameter values, combined use may result in worse (Fig. 5A) control than the better-component BCA. The overall efficacy resulting from combined use was only $\approx 3.5,1.4$, and $2.2 \%$ greater than the better component for the January, April, and July application, respectively, although statistically significant. Combined use of BCAs consistently delayed significantly the time to $10 \%$ total disease compared with the better-component BCA. This delaying effect increased with increasing model parameters (Fig. 6) and with late BCA application time: the delay to the $10 \%$ disease was $\approx 6,10$, and 17 days for the January, April, and July application, respectively.

The overall efficacy from the combined use of BCAs was significantly less than the expectation based on the Bliss independence assumption, and the difference varied with specific combinations of BCAs as well as with model parameters (Fig. 5). The efficacy was $\approx 3.9,8.9$, and $11.2 \%$ less than the expected for the January, April, and July application, respectively. As for the comparison with the better-component BCA, the overall difference was smallest when the competitive BCA had an optimum temperature at $25^{\circ} \mathrm{C}$ (Fig. 5G to I). Only for a few cases with small parameter values did the combined use of BCAs lead to better control than the expectation (Fig. 5D, E, G, and I) but the difference was very small.

Stochastic variability in temperature. The simulated random variation in hourly temperature did not result in much variation in efficacy: each of the coefficients of variation was $<4 \%$ and most of them were $<2 \%$. This lack of variability among replicate runs can be seen in Figure 7A, where the efficacy from the combined use is plotted against the expected or the better-component BCA for the April application. Only in one of the nine BCA combinations in the April application was the efficacy from the combined use similar to that expected (Fig. 7A), and this was for the combination of two BCAs, both with optima at $25^{\circ} \mathrm{C}$. For all the other combinations, combined use resulted in significantly lower efficacy than that expected (Fig. 7A). When compared with the better-component BCA, the combined use resulted in improved, reduced, or similar efficacy for four, two, and three combinations, respectively (Fig. 7A).
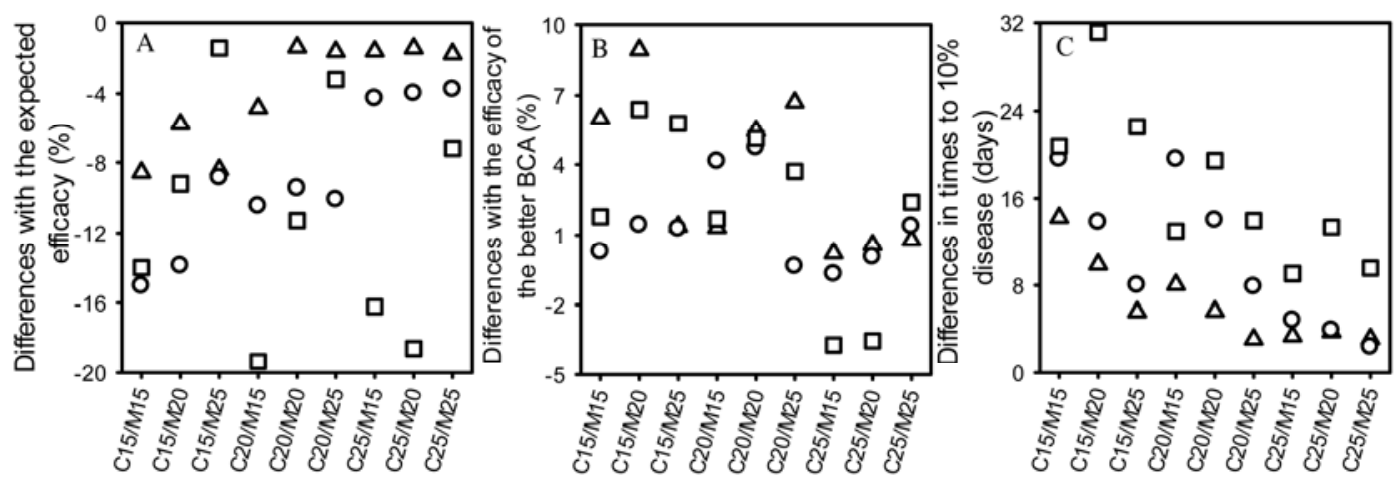

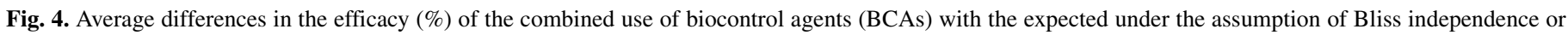

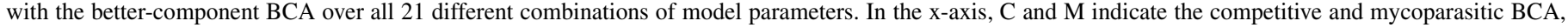

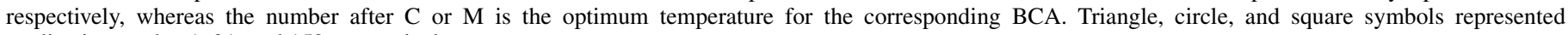
application on day 1,91 , and 152, respectively. 
For the time to $10 \%$ total disease, all combined applications of BCAs led to additional delay over the better-component BCA. Application in July led to the longest delay ( $\approx 18$ days) for the total disease to reach $10 \%$ for combined use of BCAs, compared with two other application times $(\approx 5$ days). Again, there was little variation among replicate simulations for the time to $10 \%$ total disease, which was primarily determined by the optimum temperature of the competitive BCA (Fig. 7B).

In most cases, the combined use appeared to increase the variability. There were 10 and 13 of 27 cases where the variance in the efficacy for the combined use was more than double of that of the competitive and mycoparasitic component BCA used alone, respectively. The opposite occurred in only 1 and 4 of 27 cases. There were 5 and 7 of 27 cases for which variability among replicate runs was greater for the application of a respective competitive or mycoparasitic BCA alone, respectively, than for the combined use. This proportion was significantly $<50 \%(P<0.05)$. Similar findings were obtained for the time to $10 \%$ total disease.

\section{DISCUSSION}

Previous numerical studies of a mean-field model have shown that a single BCA with multiple biocontrol mechanisms is much more effective than combined use of multiple BCAs, each with a single mechanism, regardless of the biocontrol mechanisms involved (competition, mycoparasitism, induced resistance, and antibiosis), and that combined use of two BCAs does not lead to synergistic interactions under homogeneous conditions but often leads to antagonistic interactions among two BCAs $(25,27)$. The present study investigated combined use of two BCAs under fluctuating conditions but only for the specific combination of a mycoparasitic and a competitive BCA. This particular combination combines the effect of delaying epidemic development offered by the competitive BCA with the effect of reducing overall disease (i.e., AUDPC-based efficacy measure) development offered by the mycoparasitic BCA (Fig. 3). The combined use of this particular pair of BCAs is also shown not generally to


Fig. 5. Estimated biocontrol efficacy (\%) based on area under the disease progress curve values calculated from simulated epidemics for each of the nine combinations of a mycoparasitic and a competitive biocontrol agent (BCA); the model initiated on 1 April. In total, 21 values of parameter $R$ (equation 6 ) were used for each key parameter defining a biocontrol mechanism (see text). In each graph, the upper and low axes were used to represent $R$ values for the competitive and mycoparasitic BCA, respectively. For all other parameters, default values were used (Table 1). In many cases, the biocontrol efficacy for the combined use of two BCAs overlaps that of the more efficacious one of two component BCAs. 
result in an appreciable improvement in AUDPC-based efficacy over individual BCAs applied alone under fluctuating conditions. As shown by the mean-field model $(25,27)$, in most cases, combined use resulted in lower efficacy than that expected based on the assumption of Bliss independence, indicating antagonistic interactions. This agrees with the general conclusion drawn from all published experimental studies on combined use of BCAs (24). Most of the previous claims of the existence of synergistic interactions in the combined use of two BCAs resulted from the incorrect definition of "synergy" (24).

The presence of antagonistic interactions between a competitive and a mycoparasitic BCA is surprising because there are few direct or indirect interactions in the present model. A competitive BCA is defined as a BCA that primarily colonizes healthy host tissues whereas a mycoparasitic BCA is defined as one that primarily colonizes diseased host tissues. The definition or measurement of biocontrol efficacy may partially account for the apparent antagonistic interactions between the two BCAs. The expected efficacy is calculated on the basis of AUDPC of each simulated epidemic over a period of 365 days. AUDPC is used because it captures overall disease dynamics, unlike a disease measurement at a single point such as disease incidence or severity at one particular time. A single-point efficacy is likely to be inappropriate for a biocontrol system involving mycoparasitic BCAs because of the nature of damped cyclic epidemic development (Fig. 3A). However, the exact AUDPC-based efficacy (hence, expected efficacy) also depends on the length of time used for calculating AUDPC in addition to the shape of overall epidemic development. For example, for a competitive BCA, the AUDPC-based efficacy generally decreases as the epidemic duration increases (Fig. 3A) whereas the AUDPC-based efficacy for a mycoparasitic BCA generally increases with increasing duration of epidemics (Fig. 3C). In addition to the AUDPC-based efficacy, the time to $10 \%$ total disease was also assessed in the present study. Combined use of BCA, as expected by the complementary actions of the two BCAs, resulted in longer time to $10 \%$ disease than the better-component (competitive) BCA used alone but the difference is usually small (Fig. 6). However, it is not possible to calculate the expected time to $10 \%$ for combined used of the BCAs on the basis of Bliss independence and, hence, not

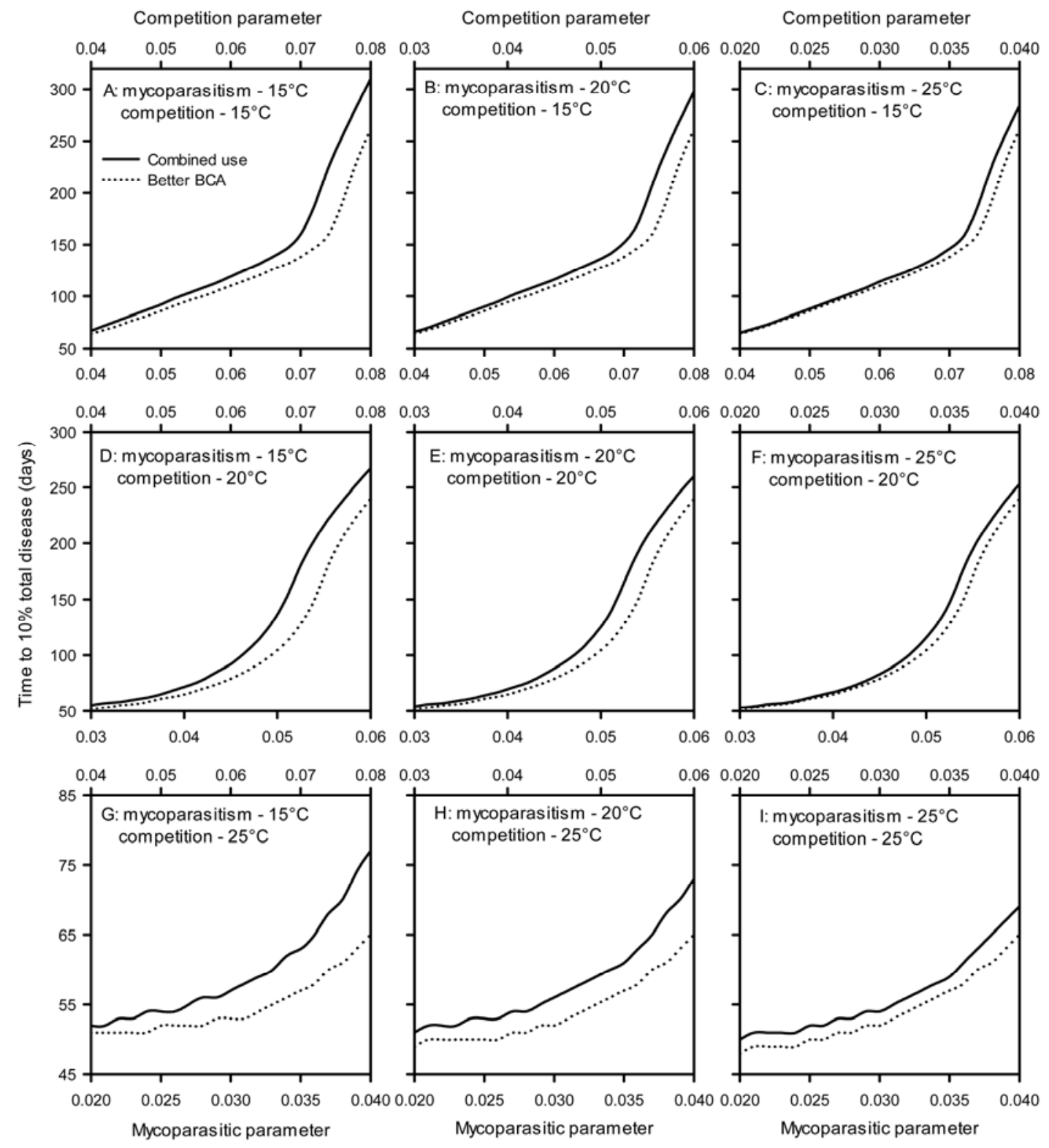

Fig. 6. Time to $10 \%$ total disease for each of the nine combinations of a mycoparasitic and a competitive biocontrol agent (BCA) when applied on 1 April. In total, 21 values of parameter $R$ (equation 6) were used for each key parameter defining a biocontrol mechanism (see text). In each graph, the upper axis and lower axis were used to represent $R$ values for the competitive and mycoparasitic BCA, respectively. For all other parameters, default values were used (Table 1 ). 
possible to assess whether there are synergistic or antagonistic interactions between the two BCAs for this particular efficacy measure. However, given the small differences in the time between the combined-use and the more efficacious BCA, synergy between the two BCAs is not likely. Appropriate quantification of efficacy for a given pathosystem needs further research.

In some cases, combined use of BCAs resulted in a lower AUDPC-based efficacy than the more efficacious (competitive) BCA used alone. This is most likely due to the difference in the initial host area assigned to the competitive BCA. For application of only a competitive BCA, $50 \%$ of host area was assigned to this BCA, compared with $37.5 \%$ in combined use of two BCAs. As defined in the present study, the initial suppression (delay) in epidemic development is primarily achieved through the competitive BCA. Thus, a lower initial colonized area for the competitive BCA may lead to a long-lasting effect on the epidemic development, particularly when the rate of BCA development is low (due to the lower $R$ parameter values or suboptimum temperature), leading to lower efficacies for combined use of BCAs than the competitive BCA used alone. However, it is not possible to circumvent this issue because we need to satisfy the requirements that (i) total host area sums to 1.0 and (ii) a single host tissue can only be in one state.

One of the main reasons for combined use of BCAs is to better exploit temporal fluctuating conditions through the use of several BCAs that are differentially adapted to different conditions. It has been speculated that this complementary adaptation to temporal conditions may lead to synergy among BCAs. However, this study shows that synergy in biocontrol is unlikely under fluctuating conditions. This could be explained by the following reasoning. First, overall biocontrol efficacy is dependent on the BCA population size as well as its ability to colonize healthy or diseased tissues. Second, during suboptimum conditions, the rate of BCA colonizing healthy or diseased tissues is lower which, combined with a constant mortality assumed in the present study (although, in real situations, the mortality likely increases during the suboptimum conditions), leads to a reduced BCA population. Finally, when the condition returns to optimum for this particular BCA, it cannot achieve the efficacy immediately before the onset of the previous suboptimum conditions because of the reduced population size. In essence, population reduction and recovery during the respective periods of suboptimum and optimum con- ditions resulted in lower efficacy than the expected based on the environmental conditions only.

The dynamics of biocontrol systems differed greatly between constant and fluctuating conditions. For the pathogen-only model, disease development was slower under fluctuating conditions than under constant conditions due to often observed nonlinear effects noted by other authors $(17,22)$. On the other hand, in the presence of a competitive BCA, disease developed more slowly under the constant temperature than under fluctuating temperature. This is most likely due to the fact that the nonlinear effect for the BCA was greater than for the pathogen: the maximum rate for the $\mathrm{BCA}$ was twice that for the pathogen. In contrast, in the presence of a mycoparasitic BCA, disease developed more slowly under fluctuating temperature than under constant temperature. This arose from the fact that the population increase of a mycoparasitic BCA is primarily through its colonization of the pathogen in diseased tissue. Therefore, the effect of fluctuating temperature on disease development in the presence of a mycoparasitic BCA is expected to be similar to the pathogen-only scenario. Therefore, relative disease development under fluctuating or constant temperature in the presence of a $\mathrm{BCA}$ depends on the relative magnitude of the nonlinear relationships of the BCA and the pathogen with environmental factors and the biocontrol mechanisms involved. The relative biocontrol performance of a single BCA and two BCAs was shown experimentally to vary greatly with the extent of temperature fluctuation (26).

Additional stochastic variation in temperature around simulated hourly temperatures did not result in much variation in biocontrol efficacy among replicated simulation runs. In contrast, in many experimental studies, large variability in biocontrol efficacy among replicated experiments has often been observed $(7,18,26)$. This difference may arise from the fact that a smooth, continuous function was used in this study to describe the effect of a fluctuating variable on the pathogen and BCA development. Thus, the effect of a small random variation in temperature on the pathogen and BCA development, as estimated by the continuous function, at any given time is minimal or negligible and likely to cancel out over the entire simulated season. However, if the effect of an environmental factor on the BCA or pathogen development is extremely nonlinear or the temporal dynamics of the underlying environmental factor is not as smooth as the temperature profile modeled in this study, then variability in biocontrol efficacy
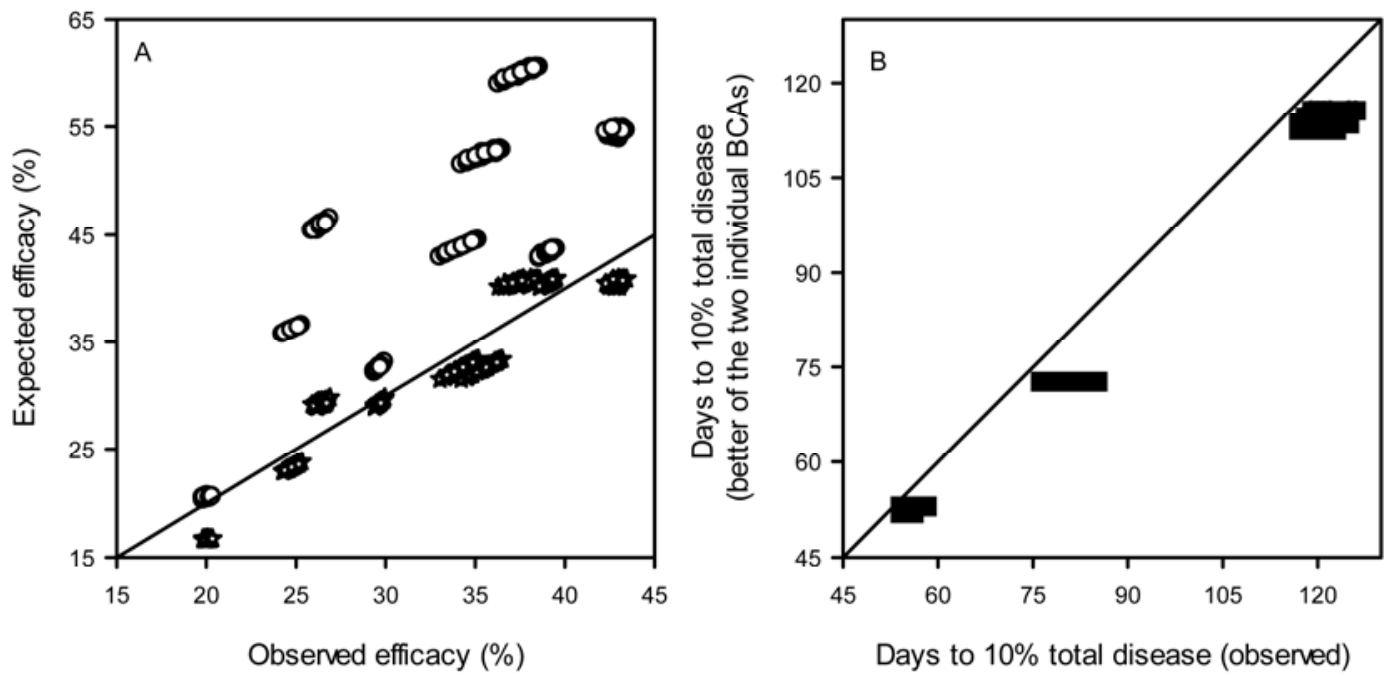

Fig. 7. Estimated biocontrol efficacy (\%) based on area under the disease progress curve values calculated from simulated epidemics for each of the nine combinations of a mycoparasitic and a competitive biocontrol agent (BCA) in all 50 replicate runs in which stochastic variability was introduced to hourly temperatures; the model initiated on 1 April. A, Efficacy of the combined use against the expected based on the Bliss independence (circle) or the efficacy of the better-component BCA (cross) and B, time to $10 \%$ total disease for the combined use against that for the better-component BCA. In B, values fell into three clusters, which correspond to the optimum temperature of the competitive component BCA: increased optimum temperatures led to a shorter time to $10 \%$ total disease. Default parameters (Table 1) were used in the model. 
(disease development) among replicate runs may be much greater. For example, for many pathogens, free water is necessary for infection, representing an extreme nonlinear relationship (yes or no). Furthermore, variability in rainfall patterns (hence, free water) is also greater than we modeled for temperature in this study. For example, in dry years, apple scab will not develop but it will rapidly spread in wet seasons, particularly during the early season. Similarly, it would be expected that development or survival of a BCA is critically dependent on moisture-related variables, potentially resulting in even greater variability in disease development.

It was reported in a few experimental studies that combined use of BCAs may lead to reduced variability among replicate experiments $(7,18)$. However, this simulation study suggested that the variability among replicate runs for more combinations of BCAs tended to be greater for the combined use of BCA than for use of individual BCAs under temporal fluctuating conditions. It is possible that incorporation of more extreme nonlinear relationships between BCA or pathogen development and fluctuating variables, as mentioned above, may alter this overall pattern. Currently, there is a scarcity of experimental data available to provide sufficient support either way regarding the variability in combined or individual use of BCAs.

In contrast to the low variability among replicate runs, there was greater variability in the biocontrol efficacy among the nine combinations of two BCAs at a given application time. There was also considerable variability in the efficacy among the three application times. Such large variability probably resulted from the fact that different combinations of BCAs (with different optimum temperatures) differed greatly in their developmental rates relative to the pathogen under a given condition. Because simulated temperatures were mostly $<23^{\circ} \mathrm{C}$, biocontrol is expected to be least effective when both BCAs had the optimum at $25^{\circ} \mathrm{C}$. On the other hand, when both BCAs had the optimum at $15^{\circ} \mathrm{C}$ and were applied earlier in the season (hence, at lower temperatures), the efficacy was greatest. These results indicate the importance of characterizing biocontrol activity in relation to environmental conditions and pathogen development. Unfortunately, apart from a few studies $(3,4,11,14)$, this aspect of biocontrol research has been neglected thus far. The present study did not consider the effect of fluctuating conditions on BCA survival because there are even fewer data available on this aspect. Therefore, for effective deployment of BCAs, it is critical to understand the relationship of BCA development and activity with environmental conditions.

Combined use of two BCAs under temporal fluctuating conditions is likely to result in antagonistic interactions rather than synergistic interactions among BCAs, although combined use may lead to a short delay in initial epidemic development. However, synergy may be expected if the two BCAs occupied different niches because, in this situation, the advantage of each individual BCA over the other under a given niche is more or less invariant over time. This is particularly the case when a pathogen is able to infect different host tissues, such as Botrytis cinerea (leaves, flowers, and fruit); however, two BCAs may preferentially occupy different host tissues. The improved efficacy from combined use of two BCAs that occupy different niches was observed for $B$. cinerea on tomato $(12,13)$. In a similar concept, combined use of two BCAs may lead to a much reduced pathogen refuge size (10). Further research is needed to establish under what spatially heterogeneous conditions the combined use of BCAs may lead to synergistic interactions and also reduced variability.

\section{LITERATURE CITED}

1. Bliss, C. I. 1956. The calculation of microbial assays. Bacteriol. Rev. 20:243-258.

2. Cunniffe, N. J., and Gilligan, C. A. 2011. A theoretical framework for biological control of soil-borne plant pathogens: Identifying effective strategies. J. Theor. Biol. 278:32-43.

3. Dik, A. J., and Elad, Y. 1999. Comparison of antagonists of Botrytis cinerea in greenhouse-grown cucumber and tomato under different climatic conditions. Eur. J. Plant Pathol. 105:123-137.

4. Elad, Y., and Kirshner, B. 1993. Survival in the phylloplane of an introduced biocontrol agent (Trichoderma harzianum) and populations of the plant pathogen Botrytis cinerea as modified by abiotic conditions. Phytoparasitica 21:303-313.

5. Elad, Y., and Stewart, A. 2007. Microbial control of Botrytis spp. In: Botrytis: Biology, Pathology and Control. Y. Elad, B. Williamson, P. Tudzynski, and N. Delen, eds. Kluwer Academic Publishers, Dordrecht, The Netherlands.

6. Freeman, S., Minz, D., Kolesnik, I., Barbul, O., Zveibil, A., Maymon, M., Nitzani, Y., Kirshner, B., Rav-David, D., Bilu, A., Dag, A., Shafir, S., and Elad, Y. 2004. Trichoderma biocontrol of Colletotrichum acutatum and Botrytis cinerea and survival in strawberry. Eur. J. Plant Pathol. 110:361370 .

7. Guetsky, R., Shtienberg, D., Elad, Y., and Dinoor, A. 2001. Combining biocontrol agents to reduce the variability of biological control. Phytopathology 91:621-627.

8. Guetsky, R., Shtienberg, D., Elad, Y., Fischer, E., and Dinoor, A. 2002. Improving biological control by combining biocontrol agents each with several mechanisms of disease suppression. Phytopathology 92:976-985.

9. Jeger, M. J., Jeffries, P., Elad, Y., and Xu, X. M. 2009. A generic theoretical model for biological control of foliar plant diseases. J. Theor. Biol. 256:201-214.

10. Johnson, K. B. 2010. Pathogen refuge: a key to understanding biological control. Annu. Rev. Phytopathol. 48:141-160.

11. Kessel, G. J. T., De Haas, B. H., Van Der Werf, W., and Köhl, J. 2002. Competitive substrate colonisation by Botrytis cinerea and Ulocladium atrum in relation to biological control of B. cinerea in cyclamen Mycol. Res. 106:716-728.

12. Le Floch, G., Tambong, J., Vallance, J., Tirilly, Y., Levesque, A., and Rey, P. 2007. Rhizosphere persistence of three Pythium oligandrum strains in tomato soilless culture assessed by DNA macroarray and real-time PCR. FEMS Microbiol. Ecol. 61:317-326.

13. Le Floch, G., Vallance, J., Benhamou, N., and Rey, P. 2009. Combining the oomycete Pythium oligandrum with two other antagonistic fungi: root relationships and tomato grey mold biocontrol. Biol. Control 50:288-298.

14. Mitchell, J. K., Jeger, M. J., and Taber, R. A. 1987. The effect of temperature on colonisation of Cercosporidium personatum leaf spot of peanuts by the hyperparasite Dicyma pulvinata. Agric. Ecosyst. Environ. 18:325-332.

15. Paulitz, T. C., and Belanger, R. R. 2001. Biological control in greenhouse systems. Annu. Rev. Phytopathol. 39:103-133.

16. Press, William H., Flannery, Brian P., Teukolsky, Saul A., and Vetterling, William T. 1989. Numerical Recipes in Pascal: The Art of Scientific Computing. Cambridge University Press, Cambridge.

17. Scherm, H., and van Bruggen, A. H. C. 1994. Global warming and nonlinear growth: how important are changes in average temperature? Phytopathology 84:1380-1384.

18. Szczech, M., and Dyki, B. 2007. Combination of microbial biocontrol agents to control Rhizoctonia damping-off and Fusarium wilt of tomato. Bull. OILB/SROP 30:415-418.

19. Whipps, J. M. 1997. Developments in the biological control of soil-borne plant pathogens. Adv. Bot. Res. 26:1-134.

20. Whipps, J. M. 2001. Microbial interactions and biocontrol in the rhizosphere. J. Exp. Bot. 52:487-511.

21. Xu, X.-M. 1996. The effects of constant and fluctuating temperatures on the length of the incubation period of apple powdery mildew (Podosphaera leucotricha). Plant Pathol. 45:924-932.

22. Xu, X.-M. 1996. On estimating non-linear response of fungal development under fluctuating temperatures. Plant Pathol. 45:163-171.

23. Xu, X.-M. 1999. Effects of temperature on the length of the incubation period of rose powdery mildew (Sphaerotheca pannosa var. rosae). Eur. J. Plant Pathol. 105:13-21.

24. Xu, X.-M., Jeffries, P., Pautasso, M., and Jeger, M. J. 2011. Combined use of biocontrol agents to manage plant diseases in theory and practice: a review. Phytopathology 101:1024-1031.

25. Xu, X.-M., Jeffries, P., Pautasso, M., and Jeger, M. J. 2011. A numerical study of combined use of two biocontrol agents with different biocontrol mechanisms in controlling foliar pathogens. Phytopathology 101:10321044.

26. Xu, X.-M., Robinson, J. D., Jeger, M., and Jeffries, P. 2010. Using combinations of biocontrol agents to control Botrytis cinerea on strawberry leaves under fluctuating temperatures. Biocontrol Sci. Technol. 20:359-373.

27. Xu, X.-M., Salama, N., Jeffries, P., and Jeger, M. J. 2010. Numerical studies of biocontrol efficacies of foliar plant pathogens in relation to the characteristics of a biocontrol agent. Phytopathology 100:814-821. 